\title{
A RARE GENETIC MUTATION PRESENTING AS SCHWACHMAN DIAMOND SYNDROME
}

\section{Dr Greeshma K. Nair* \\ Dr Sameer Pawar}

\section{Dr Uday Rajput}

\section{Dr Aarti Kinikar}

Resident doctor, Department of Paediatrics, B.J.G.M.C \& Sassoon General Hospital , Pune. *Corresponding Author

Assistant Professor, Department of Paediatrics, B.J.G.M.C \& Sassoon General Hospital, Pune.

Associate Professor, Department of Paediatrics, B.J.G.M.C \& Sassoon General Hospital, Pune.

Professor and Head, Department of Paediatrics, B.J.G.M.C \& Sassoon General Hospital, Pune.

ABSTRACT Schwachman Diamond Syndrome is an extremely rare genetic condition with autosomal recessive inheritance characterized by multisystem involvement with considerable phenotypic variation. Its epidemiology is not well studied in Asia. Only 65cases have been reported from Asia most of them from Japan . Life span is usually 35 years but those with haematological complications have reduced life expectancy. We report to you a case of 14 month old boy with complaints of chronic diarrhoea and not gaining weight with previous history of multiple admissions for pneumonia, diarrhoea transfusions for severe anaemia since last 7- 8 months. Baseline investigations were suggestive of bicytopenia initially and later progressed to pancytopenia and hence bone marrow examination was done which was suggestive of bone marrow failure together with features of malabsorption lead to suspicion of Schwachman Diamond syndrome and hence genetic study was done which showed DNAJC21 gene mutation which is a rare mutation causing Schwachman Diamond Syndrome which has not been reported before to the best of our knowledge.

Our case highlights that for diagnosis of such a rare genetic disorder requires a high index of suspicion as the cost for investigation is high and hence to come to a diagnosis on such a presentation due to its multisystem involvement is very crucial for early intervention and cure and reports a rare mutation of Schwachman Diamond Syndrome.

KEYWORDS : Schwachman Diamond Syndrome, DNAJC21 mutation, bone marrow failure, malabsorption, multisystem

Introduction:

Schwachman Diamond Syndrome is a rare genetic disorder with autosomal recessive inheritance caused by mutations in SBDS gene, SDS gene, EFLl, DNAJC21, SRP54gene causing bone marrow failure with multisystem involvement in children(1). This mutant gene protein product is involved in ribosomal biogenesis hence defective protein cause failure of pancreatic acinar development, fatty replacement of pancreatic tissue, dysfunctional haematopoeitic stem cell, accelerated apoptosis of marrow progenitors, defective marrow microenvironment. Only 65 cases have been reported from Asia most of them from Japan with only a few cases reported from India. Clinical features include hamaetological manifestations of anaemia, infections, thrombocytopenia, gastrointestinal features of malabsorption, chronic diarrhea, pancreatic exocrine insufficiency, hepatomegaly and raised liver enzymes ,skeletal abnormalities, cardiac features and others like growth hormone deficiency, hypogonadism and hypothyroidism(3). We present a 14 month old boy with clinical features and bone marrow failure type 3 a variant of Schwachman Diamond Syndrome.

Case details:

A 14 month old male child b/o non-consanguinous marriage, 2nd by order presented with c/o diarrhoea on and off since last 6 months, not gaining weight. No h/o fever, dysentry, allergy. Past $\mathrm{H} / \mathrm{o}$ repeated admissions for lower respiratory tract infections and diarrhoea and $\mathrm{h} / \mathrm{o}$ receiving transfusion once $\mathrm{l}$ month back for severe anaemia. Multiple haemograms done during previous admissions s/o pancytopenia (low platelete -- > low Hb -- > low WBC-), blood indices were normal. No H/O similar complaints in family. No significant antenatal, intranatal, post natal history. On examination poorly built, Pallor + , signs of vitamin D deficiency, no abdominal distension, no hepato-splenomegaly, no lymphadenopathy. Anthropometry suggestive of Severe Acute Malnutrition.
Systemic examination was normal.

\section{Investigations:}

Baseline Laboratory results revealed Hemoglobin- 5.1gm\%, Platelete-20000/ $\mathrm{mm}^{3}$, WBC-7400 (neutrophils 66\%, lymphocyte $28 \%$ ), PBS s/o normocytic normochromic with anisopoikilocytosis with acanthocytes and few target cells, LFT and RFT, LDH within normal limits, Stool routine and cultures were negative. CRP and blood cultures were negative.

Initially patient presented as a case of chronic diarrhoea with failure to thrive with anaemia and thrombocytopenia and h/o repeated infections and transfusion in past. We ruled out chronic infections like tuberculosis and HIV and malabsorption workup was done. Then patient started developing bleeding symptoms along with pancytopenia along with diarrhoea.

Differential diagnosis for Chronic diarrhoea with failure to thrive with pancytopenia considered were malabsorption leading to vitaminBl2 and folic acid deficiency, Immunodeficiency syndromes, Bone marrow failure syndrome(CAMT,SDS), Celiac disease.

Vitamin Bl2 and Folic acid levels were normal. Immunoglobulin levels were normal. Stool for reducing substance was trace. Stool for fat globules, calprotectin, cryptosporidium and clostridium toxin was negative. Celiac disease workup was negative. X-rays done to r/o skeletal abnormalities were normal. Serum amylase levels were normal.

I need the last oxford comma): Thus we ruled out megaloblastic anaemia, chronic infections, malabsorption, immunodeficiency disorders, and bone marrow biopsy was 
done which was suggestive of hypoplastic hypofunctioning marrow. Since immunodeficiency and malabsorption syndromes were ruled out, we decided to go ahead with genetic study (clinical exome study) which was s/o bone marrow failure syndrome type 3 which is a variant of Schwachman Diamond Syndrome in which DNAJC2lgene located on exon8 is pathogenic.

\section{Management and follow up:}

After acute infection was treated the child is currently on lactose free monosacharide diet and supplements and haemogram monitoring is done every 15 days. Since haematopoietic stem cell transplantation is the only definitive treatment for bone marrow failure in Scwachman Diamond syndrome, parents were counselled for the same and sibling's HLA typing was sent and was found to be a match and now our patient has been planned for bone marrow transplant.

\section{DISCUSSION:}

Schwachman Diamond Syndrome, first identified in 1964, is a rare genetic condition with multisystem involvement. Mutations in SBDS gene, SDS gene, EFLl, DNAJC21, SRP54.Autosomal recessive inheritance is seen. SBDS gene on long arm of chromosome7 is the most frequently mutated gene(1).This gene codes for a ribosomal protein of 250 aminio acids involved in cellular RNA metabolism or ribosome assembly or function for cellular process of TRANSLATION and is found in all tissues. At present it is unclear how disruption of basic cellular process of translation leads to the tissue and oragan specic manifestation seen in SDS such as failure of pancreatic acinar development, fatty replacement of pancreatic tissue, dysfunctional haematopoeitic stem cell, accelerated apoptosis of marrow progenitors, defective marrow microenvironment. Various clinical features are haematological like anaemia, thrombocytopenia, bone marrow failure, infections due to neutropenia, low immunoglobulin levels, and increased risk of developing myelodysplastic syndrome, leaukemia (AML)(4) '(5), gastrointestinal features like chronic diarrhoea, steatorrhea, pancreatic insufficiency(6), skeletal manifestations like abnormal development of growth plate, metaphyseal dysostosis. Cardiac features like myocardial necrosis and fibrosis on hpe, depressed ventricular contractility and diastolic dysfunctions. Other features like insulin dependent diabetes, growth hormone deficiency, hypogonadotropic hypogonadism, hypothyroidism(3) have been reported in some patients.

As the disease presents as multisystem involvement there is usually a diagnostic delay and it requires a high index of suspicion for diagnosis. Recommended workup (3) - a) DAIGNOSTIC like Marrow function- complete blood count, peripheral blood smear examination, bone marrow aspirate and biopsy, Exocrine pancreatic function- trypsinogen, amylase, Genetic testing - microarray, clinical exome study for SBDS mutation analysis (7)(approximately $90 \%$ of patients meeting clinical diagnostic criteria for SDS have mutations in SBDS gene(8)) only very few other mutations have been reported such as SDS gene, EFLl, DNAJC21. b) SUPPORTIVE like liver function tests, immune workup - Iga, IgG, IgM, radiologic workup, haematologic, endocrinology, genetic, gastroenterology consultation.

A multidisciplinary approach is required for treatment and follow up of this patients with a paediatrician, haematologist, gastroenterologist, endocrinologist, nutritionist etc. Only definitive therapy for marrow failure, myelodysplastic syndrome or leukaemia in Schwachman Diamond syndrome is haematopoietic stem cell transplantation. But the success of this procedure depends on the extent of other systemic involvement like pancreatic insufficiency, cardiac involvement, endocrine involvement etc. some of which has no definite therapy. Supportive measures like haemogram monitoring and transfusions, Pancreatic Enzymes, G-CSF and dietary modification(3) are needed in most of the cases.
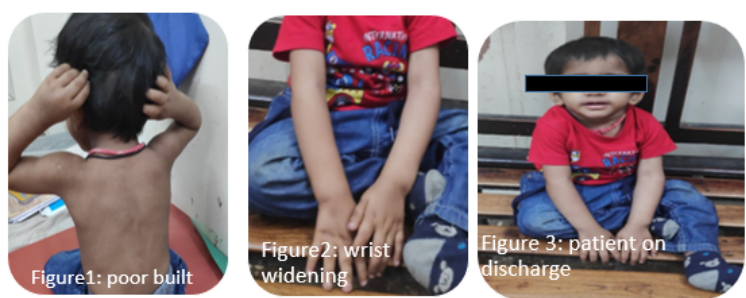

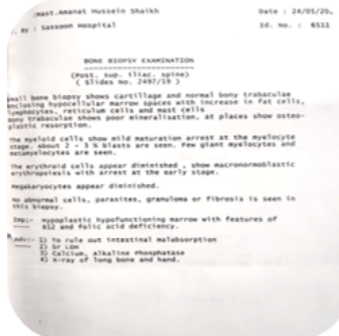

Figure 4: bone marrow biopsy report

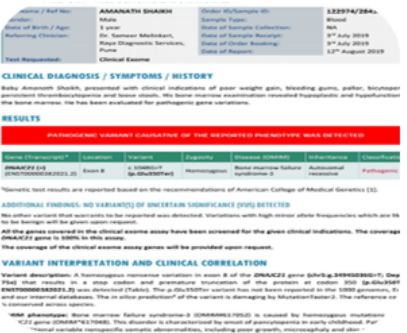

Figure 5: clinical exome study report

\section{CONCLUSION}

Schwachman Diamond Syndrome is a rare genetic disorder which requires a high index of suspicion for diagnosis, due to its multisystem involvement, to avoid diagnostic delays and as the cost for investigation is high, to come to a diagnosis on such a presentation, is very crucial for early intervention and cure. DNAJC21 gene mutation is a rare cause of Schwachman Diamond syndrome as seen in our case.

Acknowledgements:

1. Dr. S Melinkeri, Dept. Haematoncology, Sassoon hospital, Pune.

2. Dr. R Manchanda, Pathologist, Hemotech laboratory, Pune.

3. Dr. Bavdekar, Gastroenterologist, KEM hospital, Pune.

4. MEDGENOME Labs Ltd., Bangalore.

\section{REFERENCES:}

1. Ong SY, Li ST, Wong GC, Ho AYL, Nagarajan C, Ngeow J. Delayed diagnosis of Shwachman diamond syndrome with short telomeres and a review of cases in Asia. Leuk Res Reports [Internet]. 2018;9(April):54-7. Available from: https://doi.org/10.1016/i.lrr.2018.04.002

2. Brown SMN, Buchdahl R. Shwachman-Diamond syndrome in a child presenting with cystic fibrosis-type symptoms and a false-positive sweat test. J R Soc Med Suppl. 2008;101(SUPPL. 1):39-44.

3. Burroughs L, Woolfrey A, Shimamura A. Shwachman-Diamond Syndrome: A Review of the Clinical Presentation, Molecular Pathogenesis, Diagnosis, and Treatment. Hematol Oncol Clin North Am. 2009;23(2):233-48.

4. Smith OP, Hann IM, Chessells JM, Reeves BR, Milla P. Haematological abnormalities in Shwachman-Diamond syndrome. Br J Haematol. 1996;94(2):279-84.

5. Mourad S, Bilodeau M, Roussy M, Laramée L, Boulianne L, Rouette A, et al. $\mathrm{IDHl}$ as a cooperating mutation in aml arising in the context of shwachmandiamond syndrome. Front Oncol. 2019;9(AUG):1-7.

6. Mack DR, Forstner GG, Wilschanski M, Freedman MH, Durie PR. Shwachman syndrome: Exocrine pancreatic dysfunction and variable phenotypic expression. Gastroenterology. 1996;111(6):1593-602.

7. Khan AW, Minelli A Frattini A, Montalbano G, Bogni A, Fabbri M, et al. Microarray expression studies on bone marrow of patients with ShwachmanDiamond syndrome in relation to deletion of the long arm of chromosome 20 other chromosome anomalies or normal karyotype. Mol Cytogenet. 2020;13(1):1-9.

8. Zhao M, Kanegane H, Ouchi K, Imamura T, Latour S, Miyawaki T. A novel XIAP mutation in a Japanese boy with recurrent pancytopenia and splenomegaly. Haematologica. 2010;95(4):688-9. 